\title{
Estudo comparativo entre os fios de ácido poliglicólico e poliglactina na ileocistoplastia em cães (Canis familiaris)
}

\section{A comparison of polyglycolic acid and polyglactin suture in ileocystoplsty in dog (Canis familiaris)}

\author{
Maria de Lourdes Gonçalves Ferreira, ${ }^{*}$ Marília Botelho de Oliveira Chaudon, ${ }^{* *}$ Edmundo Jorge Abílio, ${ }^{* * *}$ Eulógio Carlos \\ Queiroz de Carvalho, ${ }^{* * *}$ Nelson Jamel, ${ }^{* * * *}$ Mario Antônio Pinto Romão, ${ }^{* *}$ Viviane Alexandre Nunes ${ }^{\star * * * * *}$
}

\begin{abstract}
Resumo
O presente estudo visa comparar os fios de ácido poliglicólico e de poliglactina 910 em ileocistoplastia em cães. Utilizaramse 24 cadelas, SRD (sem raça definida), adultas, pesando em média $15 \mathrm{~kg}$, divididos em dois grupos iguais, os quais foram submetidos a ileocistoplastia. No grupo A empregou-se o fio de poliglactina 910 e no B o fio de ácido poliglicólico. Os animais

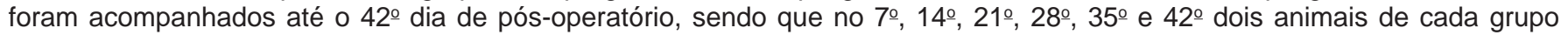
foram necropsiados para retirada da bexiga visando análise histopatológica da linha de sutura. Estipulou-se escores de 0 a 3 para reação inflamatória e absorção e de 1 a 3 para fibrose (cicatrização). Esses dados foram submetidos atratamento estatístico (teste $x^{2}-$ Qui-quadrado) onde $a=0,05$. Nos animais do grupo A, do 7으 ao 42 dia observou-se reação inflamatória compatível com o período, enquanto os cães do grupo B, além da reação inflamatória, demonstravam também presença de supuração até o 35o dia $(\mathrm{p}<0,05)$. Os cães do grupo $A$ apresentaram sinais progressivos de fibrose, a partir do 70 dia, enquanto os cães do grupo B só vieram a apresentar tais sinais após 21 dias. No 210 dia os cães do grupo B demonstraram sinais de início do processo de hidrólise, e no grupo A estes sinais só foram evidenciados no 28 dia. Os cães do grupo B apresentaram sinais de calcificação na linha de sutura a partir do 21 ㅇ dia, fato não observado em todo período nos animais do grupo A. Houve diferença significativa entre os fios utilizados no grupo $A$ e no grupo $B$, no que se refere a reação inflamatória, grau de fibrose e calcificação na linha de sutura, caracterizando o fio de poliglactina 910 como mais indicado para enterocistoplastia em cães.
\end{abstract}

Palavras-chave: estudo comparativo, ácido poliglicólico, poliglactina 910, transplante autólogo - veterinária, bexiga - cirurgia, íleo - cirurgia, cães, cicatrização, suturas.

\begin{abstract}
A comparison of polyglycolic acid and poliglactin 910 suture was performed in dogs ileocystoplasty procedures. Twenty four mongrel adult bitches weighting $15 \mathrm{Kg}$ where dividided into two similar groups and submitted to ileocystoplasty. Polyglactin was employed in group A; and Polyglycolic acid in group B. The animals were observed until $42^{\text {nd }}$ day immediate postoperative period, and two animals of each group necropsied to extract the urinary bladder for histopathological analysis. A score of zero to three represented inflammatory reaction and absortion; a score of one to three for fibrosis (cicatrization). Data statistic analysis were done ( $x^{2}$ test square - Qui) where $a=0,05$. Animals of group A showed up inflammatory reaction compatible to the period from $7^{\text {th }}$ to $42^{\text {nd }}$ day; animals of group B showed up inflammatory reaction also but suppuration till the $35^{\text {th }}$ day $(p<0,05)$. Group A dogs presented progressive signals of fibrosis form up the $7^{\text {th }}$ day; group $B$ dogs showed up beginning process after 21 days. Group B animals presented hydrolyses process in $21^{\text {st }}$ day; in group A these signals appeared in $28^{\text {th }}$ day. Animals of group B presented calcification process in the suture line from $21^{\text {st }}$ day; this was not observed in animals of group A. Showed up significative difference between suture used in group A and Group B animals, due to inflammatory reaction, fibrosis level and calcification of the suture line. We concluded that polyglactin was the most valuable for enterocystoplasty in dogs.
\end{abstract}

Keywords: comparative study, polyglycolic acid, poliglactin 910, autologo transplant - veterinary, bladder - surgery, ileo- surgery, dogs, healing, sutures.

\footnotetext{
* Professora Dra. da Disciplina de Patologia e Clínica Cirúrgica Veterinária da UFF.

** Professor Dr. do Departamento de Patologia e Clínica Veterinária da Faculdade de Veterinária UFF.

*** Professor Dr. da Disciplina de Patologia e Clínica Cirúrgica Veterinária da UENF.

**** Professor Dr. de Anatomia Patológica da Faculdade de Veterinária da UENF.

${ }^{* * * * *}$ Professor Dr. do Departamento de Cirurgia da Faculdade de Medicina da UFRJ.

${ }^{* \star * \star * *}$ Professora Ms. da Disciplina de Patologia e Clínica Cirúrgica Veterinária da UFF.
} 


\section{Introdução}

Somente com a industrialização no último século é que foram desenvolvidos novos materiais de sutura, tais como o ácido poliglicólico e a poliglactina 910. De acordo com a resposta que cada um deles produzia no organismo, puderam ser classificados em dois grandes grupos: fios absorvíveis e inabsorvíveis. Tal classificação baseia-se no tempo que cada fio leva para perder sua força tênsil e sofrer degradação pelo organismo (Edlich et al., 1987).

Os estudos utilizando enterocistoplastia realizados por Vordermark et al. (1992) e Gonzales et al. (1995) não enfocam, respectivamente, o comportamento dos fios à base de ácido poliglicólico e poliglactina e, no momento, não há uma padronização em relação ao fio de sutura ideal para ser usado neste tipo de procedimento. O melhor fio é aquele que, com a menor reação inflamatória possível, seja capaz de manter sua força tênsil até que a cicatrização da ferida cirúrgica esteja completa, e seja absorvido, evitando com isso a formação de cálculos.

O poder de recuperação da bexiga em todas as suas túnicas é notado pelos autores de forma geral, tendo, contudo, merecido atenção de Hastings et al. (1975). Utilizando fio de poliglactina, constataram que entre 14 e 21 dias do ato operatório ocorreu $100 \%$ de recuperação das estruturas da bexiga. Ellison (1996), nas mesmas condições, acrescenta a recuperação de $100 \%$ da força tênsil do órgão. Peacock (1984) afirmou que a cistectomia com mais de $75 \%$ do volume da bexiga pode ser praticada, porém, com a preservação do trígono vesical, ela se regenera em todas as funções, devido à sua grande capacidade recuperadora.

Segundo os fabricantes, o Vycril é um material de sutura absorvível confeccionado a partir de polímeros que são inertes, não-antigênicos, não-pirogênicos e provocam somente uma leve reação tissular durante a absorção (Ethicon, Johnson e Johnson), enquanto que o Safil é caracterizado como estéril, absorvível, trançado e produzido a partir de ácido poliglicólico, afirmando ainda que quando utilizado, há leve reação inflamatória que é típica de uma reação a um corpo estranho, sendo envolvido por tecido conjuntivo fibroso com o passar do tempo, até a sua absorção (B. BRAUN ${ }^{\circledR}$ ).

Fossum (2002), preferiu material de sutura absorvível para cirurgias vesical e uretral de pequenos animais, entre eles, o ácido poliglicólico e a poliglactina 910 citando que a maioria dos fios de sutura parece perder a força tênsil mais rapidamente em urina alcalina que em urina ácida infectada ou estéril.

Fontes e Sadi (1995) realizaram estudo comparando o comportamento de seis tipos de fios absorvíveis (catgut cromado, ácido poliglicólico, poliglactina 910, polidioxane, poligliconato e poliglecaprone 25) em bexiga de cão e para isso coletaram biópsias da área suturada no $7^{\circ}, 21^{\circ}, 90^{\circ}$ e $180^{\circ}$ dia pósoperatório, onde avaliaram através da histopatologia o grau de inflamação, presença de restos de fio e calcificações no local da sutura. No sétimo dia foi possível identificar macroscopicamente todos os fios de sutura na luz da bexiga; porém, com 21 dias o epitélio cobria totalmente. Microscopicamente, aos 21 dias o material de sutura era visível em todos os cães, enquanto aos 90 dias não se observava presença dos fios de ácido poliglicólico e poliglactina. A reação inflamatória dos fios foi semelhante nos intervalos estudados, não havendo diferença significativa entre eles. No sétimo dia foi encontrada uma reação inflamatória exsudativa às custas de poucos leucócitos polimorfonucleados e alguns histiócitos e linfócitos, com edema discreto. Na avaliação do $21^{\circ}$ dia foi constatada presença de grande quantidade de fibroblastos e fibrócitos, além da deposição de fibras colágenas. O urotélio já estava totalmente recomposto, mostrando cinco a dez camadas de células. A reação inflamatória foi predominantemente mononuclear com presença de histiócitos, linfócitos e raros plasmócitos distribuídos difusamente, além de cistos formados por aglomerado de células, sem no entanto caracterizar a presença de células gigantes tipo corpo estranho. Observaram ainda a presença de fibrose leve ou moderada quando analisaram o fio de poliglactina.

Gonzales et al. (1995) realizaram enterocistoplastia seromuscular em cães utilizando fio de poliglactina $910 \mathrm{na}$ sutura interrompida para reforçar sutura primária contínua com catgut. Sacrificaram os animais oito semanas após a cirurgia e ao exame post mortem observaram presença de pedículo nutridor do enxerto intacto, e este, viável histologicamente.

Boyd et al. (1994) reconstituíram 11 pacientes utilizando como material de sutura fios de ácido poliglicólico, ora utilizado em padrão contínuo, ora em sutura circular para confecção de esfíncter uretral artificial, não tendo descrito nenhum caso de deiscência ou infecção no pós-operatório.

O objetivo deste estudo comparativo foi avaliar a utilização dos fios de ácido poliglicólico e de poliglactina $910 \mathrm{em}$ ileocistoplastia em cães, e estudar a reação tecidual através do grau de inflamação, fibrose da ferida cirúrgica (cicatrização), com o intuito de verificar qual dos fios testados está mais indicado para realização deste procedimento.

\section{Material e método}

Vinte e quatro cadelas, adultas, pesando em média $15 \mathrm{~kg}$, sem raça definida (SRD), doadas pelo Centro de Controle de Zoonoses do Município de Niterói, RJ e provenientes de apreensões, foram submetidas ao experimento segundo as normas do Colégio Brasileiro de Experimentação Animal (COBEA 2003), sendo divididas em dois grupos de 12 animais cada: 0 primeiro grupo (A) submetido a implante de autoenxerto ileal, não-tubular, com pedículo vascular (ileocistoplastia) utilizando sutura em padrão contínuo tipo "Cushing" com fio poliglactina $9103.0^{2}$ sertix gastrintestinal com agulha cilíndrica $3 / 8$ de círculo medindo $3 \mathrm{~cm}$. O segundo grupo (B) diferiu do primeiro em relação ao fio utilizado, que foi de ácido poliglicólico $3.0^{3}$ sertix com agulha cilíndrica 1/2 círculo medindo $2 \mathrm{~cm}$.

Foram realizados exames radiográficos simples e contrastados e ultra-sonográfico para avaliar a impermeabilidade da bexiga e EAS (Elementos Anormais e Sedimentoscopia) para determinar o pH da urina no $7^{\circ}, 14^{\circ}, 21^{\circ}, 28^{\circ}, 35^{\circ}$ e $42^{\circ}$ dias.

A cada sete dias, do 70 até o $42^{\circ}$ dia, dois animais de cada grupo foram eutanasiados, através de indução anestésica de ação central (coma barbitúrico). Na necroscopia, as bexigas foram colhidas in totum, preenchidas e mergulhadas em formalina neutra tamponada a $10 \%$. Após a fixação, as peças foram clivadas, com atenção para o enxerto, os limites da ferida e a área de sutura e reidratadas em soluções aquosas alcoólicas, de concentrações crescentes, diafanizadas em xilol, embebidas e incluídas em parafina histológica. Os cor- 
tes de 5 micra foram efetuados em micrótomo Spencer, da América Optical. A coloração foi a de rotina, pelo método de hematoxilina e eosina, segundo Prophet et al. (1994), e a microscopia se deu em videomicroscópico de luz (Olympus BX50), com sistema automático de fotomicrografia (Olympus PM 30).

Para determinação do grau de inflamação, fibrose e processo de absorção foi utilizado um sistema de escore: a) para a calcificação na linha de sutura e formação de cálculo vesical foi considerado presença e ausência; b) reação inflamatória: 0 = reação inflamatória ausente; 1 = reação inflamatória aguda; 2 = reação inflamatória crônica e 3 = reação inflamatória com supuração; c) grau de fibrose: (1) = primeiro estágio de granulação; (2) = fibrose incipiente; (3) = fibrose densa e d) processo de absorção: 0 = Ausência de sinais de absorção; 1 = presença de células gigantes com fio fagocitado; 2 = presença de células gigantes em fagocitose com sinais de início de hidrólise e 3 = dissolução total do fio. Os dados são apresentados por meio de estatística descritiva, com quadros e gráficos, bem como a estatística inferencial, por meio do teste $x^{2}$ (Qui-quadrado). O nível de significância adotado foi de $5 \%(a=0,05)$.

\section{Resultados e discussão}

Sharma (1977); Sharma e Khan (1978); Salle et al. (1990); Fries et al. (1991); Gonzales et al. (1995) e Nandi et al. (1996) utilizaram dois planos de sutura para fixação do enxerto em enterocistoplastia. Diferentemente destes autores, no presente estudo utilizou-se o plano de sutura contínuo único, o qual mostrou-se eficiente para fixação do enxerto conferindo vedação hermética à bexiga, comprovada pelo teste do soro proposto por Slatter (1998), conforme também empregado por Vordermark et al. (1992); e Boyd et al. (1994), enquanto que Slatter (1998) recomendou o padrão de sutura de Cushing sobreposta por Cushing ou Lembert no reparo de ruptura de bexiga, relatando que em cães sadios não há diferença entre o padrão contínuo único ou em dupla camada.

Desgrandchamps e Griffith (1999) afirmaram que uma bexiga recuperada cirurgicamente deve promover armazenamento adequado, permitir eliminação completa da urina e preservar a função renal, conforme também foi observado no presente estudo, onde todos os cães, tanto do grupo A como do B, não exibiram evidências de quaisquer alterações funcionais no trato urinário tais como extrusão do enxerto, infecção ou formação de cálculo. Estes resultados diferem de Salle et al. (1990), que cogitaram a hipótese de isquemia, contração ou severa reação inflamatória provocada por infecção ou irritação química pelo contato do enxerto com a urina. Também não foram observadas todas das alterações citadas por Laus et al. (1995) em relação a enteroanastomose, onde os animais do presente experimento apresentaram evolução clínica satisfatória, não sendo observandos sinais de distensão abdominal relatados pelos autores, mas sim alguns animais de ambos os grupos manifestaram prostração e febre, tendo-se restabelecido normalmente após 24 horas, tornandose ativos e com apetite, o que está de acordo com Salle et al. (1990) e Gonzales et al. (1995), que consideraram comum a ocorrência de febre nas primeiras 72 horas. Outro aspecto digno de nota é que todos os animais iniciaram a micção de 12 a 18 horas após a cirurgia, a qual se mostrou sem nenhuma alteração, enquanto a exoneração intestinal ocorreu após
24 horas, como também observado por Laus et al. (1995) em relação ao trânsito intestinal após enteroanastomoses e por Vordermark et al. (1992), que associaram o esvaziamento vesical e menor tempo de contato da urina com a mucosa intestinal à ausência de distúrbios eletrolíticos. Já no experimento de Salle et al. (1990) os cães, apesar de urinarem espontaneamente, apresentaram muco na urina após a enterocistoplastia.

Kaminsky et al. (1978), realizaram sutura com fio de ácido poliglicólico na bexiga de coelhos, cães e gatos e só encontraram cálculos nos coelhos; Fontes e Sadi (1995) atribuíram a não formação de cálculo ao pH ácido da urina dos cães; os resultados obtidos corroboram esta observação, visto que todos os animais do presente estudo apresentaram durante o período de acompanhamento, $\mathrm{pH}$ urinário ácido variando entre 6,3 e 6,5. Estes autores ressaltaram ainda que a ausência de contato direto do fio com a urina também dificulta a formação de cálculos. O padrão de sutura de Cushing utilizado aqui, não permitiu o contato do fio com a urina e não foram encontrados urólitos. Entretanto, os animais do grupo B apresentaram calcificação na linha de sutura (Figura 1) a partir do $21^{\circ}$ dia, evoluindo para metaplasia óssea no $42^{\circ}$ dia pós-operatório, contradizendo Fontes e Sadi (1995), que consideraram o cão como modelo impróprio para o estudo da calcificação na linha de sutura. Por outro lado, Fossum (2002) recomendou o uso de fios de ácido poliglicólico e de poliglactina 910, ressaltando que causam menor reação inflamatória, são mais resistentes em urina ácida estéril ou infectada e promove menor formação de cálculo que os fios inabsorvíveis. Entretanto, não faz referência à calcificação na linha de sutura, além de nossos resultados não serem homogêneos para os dois fios, tendo o fio de ácido poliglicólico provocado mais reação inflamatória e calcificação na linha de sutura.

Uma bexiga recuperada cirurgicamente, além de manter suas funções fisiológicas conforme postulam Desgrandchamps e Griffth (1999), também deve, dentro do possível, restaurar e/ ou preservar sua arquitetura anatômica. As imagens radiográficas e ultra-sonográficas do 70 ao $21^{\circ}$ dias, mostraram pequenas alterações na conformação anatômica das bexigas situadas na face da enxertia, em ambos os grupos, desaparecendo, porém, a partir do 28 dia. Desta forma, além da manutenção das funções fisiológicas, preservou-se a anatomia do órgão; o emprego do diagnóstico por imagem é citado por alguns autores, como Gonzalez et al. (1995), que trabalharam com pacientes humanos; entretanto, não relatam os resultados obtidos. Também não foi detectada, em todo o período, nenhuma evidência de ruptura da sutura, confirmando o que Bourne et al. (1988) revelaram a respeito da manutenção da força tênsil dos fios estudados até o 14ํ dia.

Fontes e Sadi (1995), encontraram no 7o dia reação inflamatória exsudativa, caracterizada por poucos polimorfonucleares e alguns mononucleares com presença de edema discreto semelhantes para os fios estudados. No presente estudo, o grupo A apresentou o mesmo padrão de reação inflamatória com ausência de edema; já o grupo B evidenciou, além da reação inflamatória, derrame hemorrágico e supuração em alguns pontos (Figura 3), o que pode estar associado ao fio de ácido poliglicólico produzir reação inflamatória exsudativa tipo corpo estranho mais intensa que a descrita pelo fabricante do mesmo, o que retardou a cicatrização. De certo modo, estas observações contradizem Postlethwait et al. (1975) e 
Fontes e Sadi (1995), que associaram a reação até o 70 dia ao trauma provocado pela passagem da agulha e não ao fio. Entretanto, deve ser considerado que estes resultados referem-se a animais sadios, devendo ser realizadas observações em pacientes portadores de afecção vesical para meIhor elucidar este comportamento dos fios testados.

No $14^{\circ}$ dia, o grupo A revelou material fibrocelular com reação inflamatória aguda leve e o grupo $\mathrm{B}$ persistência da reação inflamatória e presença de granuloma piogênico (Quadro 1). Fontes e Sadi (1995), constataram que o processo de regeneração tecidual ocorre simultaneamente ao processo inflamatório, tendo encontrado polimorfonucleares e fibroblastos. No presente estudo, observou-se regeneração tecidual no grupo $A$, enquanto o grupo $B$ revelou reação inflamatória com processo piogênico (Quadro 1).

Bimonte (1997) citou que a velocidade de absorção está diretamente relacionada com a vascularização do tecido e que a poliglactina 910 é mais resistente à hidrólise que o ácido poliglicólico, fato que também observamos no 210 dia. O grupo $B$ apresentou sinais de hidrólise, fato observado no grupo A, somente no 28 dia (Quadro 2).

Fatureto e Teixeira (1993) e Fontes e Sadi (1995), afirmaram que a forma de absorção determina o grau de reação inflamatória, mas apesar dos fios utilizados no experimento sofrerem hidrólise (Quadro 2), a reação ao ácido poliglicólico foi mais intensa que a da poliglactina.

No grupo B notou-se presença de tecido cicatricial (granulação) somente no $35^{\circ}$ dia, mas Fontes e Sadi (1995) afirmaram que o processo cicatricial ocorre concomitantemente ao processo inflamatório. A partir da observação dos dados referentes

Quadro 1 - Escores da reação inflamatória e da fibrose (valores entre parênteses) dos cães do grupo A (poliglactina) e B (ácido poliglicólico), sendo 2 animais por dia para cada grupo, aos 7, 14, 21, 28, 35 e 42 dias após a operação.

\begin{tabular}{|c|c|c|c|c|c|c|}
\hline DIAS & $\mathbf{7}^{\mathbf{0}}$ & $\mathbf{1 4}^{\mathbf{0}}$ & $\mathbf{2 1}^{\circ}$ & $\mathbf{2 8}^{\circ}$ & $\mathbf{3 5}^{\circ}$ & $\mathbf{4 2}^{\circ}$ \\
\hline \multirow{2}{*}{ GRUPO A } & $1(2)$ & $1(2)$ & $2(2)$ & $0(2)$ & $0(3)$ & $0(3)$ \\
\cline { 2 - 7 } & $1(2)$ & $1(2)$ & $2(2)$ & $0(2)$ & $0(3)$ & $0(3)$ \\
\hline \multirow{2}{*}{ GRUPO B B } & $3(1)$ & $3(1)$ & $3(2)$ & $3(2)$ & $3(2)$ & $2(2)$ \\
\cline { 2 - 7 } & $3(1)$ & $3(1)$ & $3(2)$ & $3(2)$ & $3(2)$ & $2(2)$ \\
\hline
\end{tabular}

Escores de reação inflamatória: 0 = reação inflamatória ausente; 1 = reação inflamatória aguda; 2 = reação inflamatória crônica e 3 = reação inflamatória com supuração. Escores de grau de fibrose: $(1)=$ primeiro estágio de granulação; (2) = fibrose incipiente; (3) = fibrose densa.

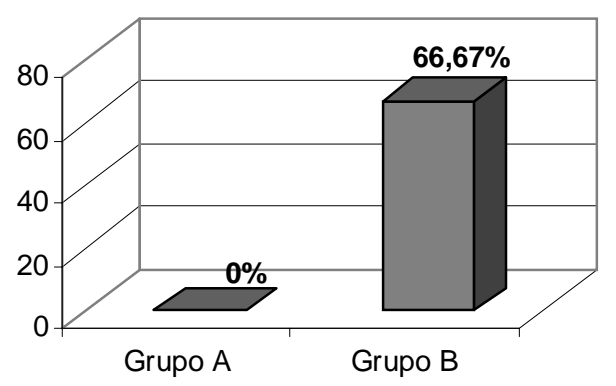

Figura 1 - Gráfico de Percentagens de animais que apresentaram calcificação nos grupos A e B. ao grau de inflamação e fibrose no presente estudo, do $7^{\circ}$ ao $42^{\circ}$ dia, pode-se afirmar que quanto mais intensa for a reação inflamatória, mais lento será o processo de fibrose, ou seja, a cicatrização da ferida cirúrgica (Figura 2).

Atribuiu-se o retardo do processo cicatricial do grupo B (Figura 2) à intensa reação inflamatória, caracterizada por reação inflamatória supurativa, fator complicador para cicatrização como também relataram Ellison (1996), Slatter (1998) e Harari (1999).

Fontes e Sadi (1995), caracterizaram a reação inflamatória no $21^{\circ}$ dia como predominantemente mononuclear, fato observado no grupo A; já o grupo $B$, evidenciou sinais de reação inflamatória com supuração até o 35 dia (Quadro 1 e Figura 3).

Rodeheaver et al. (1983) citaram que os fios de poliglactina e de ácido poliglicólico tiveram melhor aceitação com menos riscos e inconvenientes quando a indústria passou a produzilos encapados. No experimento em tela, utilizaram-se fios encapados e, mesmo assim, a reação inflamatória ao fio de ácido poliglicólico foi mais intensa e desfavorável histologicamente que a apresentada pelo fio de poliglactina com predominância de reação inflamatória com supuração (Quadro 1) e calcificação na linha de sutura (Figura 2).

O teste c2 (Qui-quadrado) aplicado ao Quadro 1 para testar a hipótese de independência dos fatores ao nível de $5 \%$ de probabilidade $(a ́=0,05)$, com o valor de $\mathrm{c} 2$ calculado $=17,14$ demonstrou que a reação inflamatória nas suturas depende do tipo de fio utilizado $(p<0,05)$. Sendo assim, quando se utiliza a poliglactina 910 , a percentagem de reação inflamatória é inferior à percentagem de reação inflamatória relativa ao ácido poliglicólico $(p<0,05)$.

Quadro 2 - Escores de processo de absorção dos cães do grupo A (poliglactina) e B (ácido poliglicólico), sendo 2 animais por dia para cada grupo, aos $7,14,21,28,35$ e 42 dias após a operação.

\begin{tabular}{|c|c|c|c|c|c|c|}
\hline DIAS & $\mathbf{7}^{\mathbf{0}}$ & $\mathbf{1 4}^{\mathbf{0}}$ & $\mathbf{2 1}^{\mathbf{0}}$ & $\mathbf{2 8}^{\mathbf{0}}$ & $\mathbf{3 5}^{\mathbf{0}}$ & $\mathbf{4 2}^{\mathbf{0}}$ \\
\hline \multirow{2}{*}{ GRUPO A A } & 0 & 1 & 1 & 2 & 3 & 3 \\
\cline { 2 - 7 } & 0 & 1 & 1 & 2 & 3 & 3 \\
\hline \multirow{2}{*}{ GRUPO B } & 1 & 1 & 2 & 2 & 3 & 3 \\
\cline { 2 - 7 } & 1 & 1 & 2 & 2 & 3 & 3 \\
\hline
\end{tabular}

Escore de processo de absorção: 0 = Ausência de sinais de absorção; 1 $=$ presença de células gigantes com fio fagocitado; 2 = presença de células gigantes em fagocitose com sinais de início de hidrolise e $3=$ dissolução total do fio.

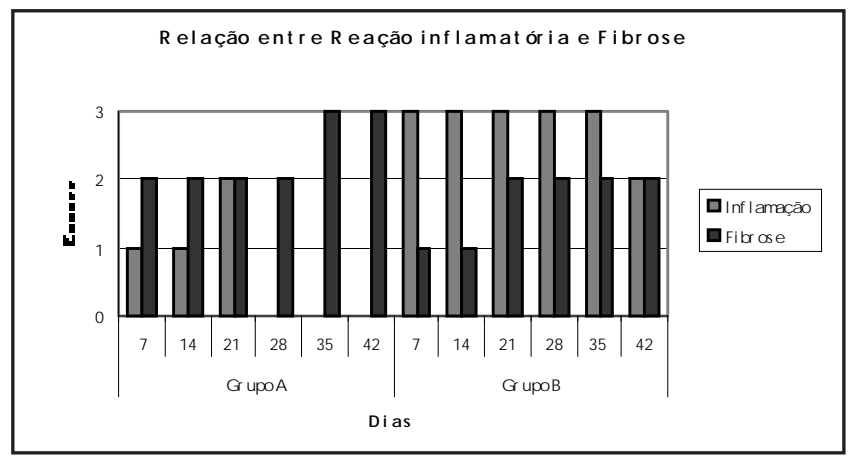

Figura 2 - Gráfico relacionando o grau de inflamação com a fibrose na linha de sutura no $7^{\circ}, 14^{\circ}, 21^{\circ}, 28^{\circ}, 35^{\circ}$ e $42^{\circ}$ dias, nos animais dos grupos A (poliglactina) e B (acido poliglicólico). 


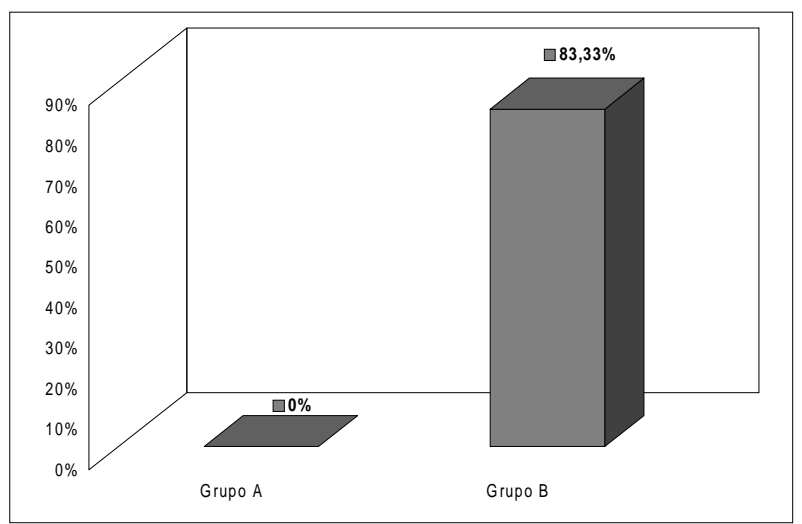

Figura 3 - Gráfico de Percentagens de animais que apresentaram reação inflamatória com supuração nos grupos A e B.

\section{Referências}

BIMONTE,D. Reacciones de materiales de sutura usados en cirugía veterinaria. Veterinaria, v. 33, n. 133, enero/marzo, p. 12-14, 1997.

BOURNE, R. B.; BITAR, H.; ANDREAE, P. R. et al. In vivo caparison of four absorbable sutures: Vycril, Dexon plus, Maxon and PDS. Canadian Journal Surgery, v. 31, n. 1, p. 43-45, 1988.

BOYD, S. D.; ESRIG, D.; STEIN, J. P; FREEMAN, J. A.; SKINNER,D. G. Undiversion in men following prior cystoprostectomy and cutaneous diversion. Is it practical? Journal Urology, v. 152, august, p. 334-337, 1994.

COLÉGIOBRASILEIRODE EXPERIMENTAÇÃO(COBEA). Princípios éticos na experimentação animal. Disponível em:(http://www.meusite. com.br/COBEA/etica.html) Acesso em 13 de junho de 2003.

DESGRANDCHAMPS, F.; GRIFFITH, D. P. The artificial bladder. Europe Urology, v. 35, n. 4, p. 257-66, April, 1999.

EDLICH, R. F.; RODEHEAVER, G. T.; THACKER, J. G. Considerationasin the choice of sutures for wound closure of the genitourinary tract. Journal Urology, v. 137, p. 373-379, 1987.

ELLISON, G. W. Cicatrização visceral e distúrbios decorrentes da reparação. In: BOJRAB, M. J. Mecanismos da moléstia na cirurgia de pequenos animais. 2. ed., São Paulo: Manole, 1996, 1v., Cap. 1, p. 2-8.

FONTES, M. A. Q. R.; SADI, M. V. Estudo experimental comparativo com fios de sutura absorvíveis em bexiga de cães. Revista do Colégio Brasileiro de Cirurgiões. v. 23, n. 2, p. 83--88, 1995.

FATURETO, M. C.; TEIXEIRA, V. P. A. Reação inflamatória a fios de sutura. In: HERING, F. L. O.; GABOR, S.; ROSENBERG, D. Bases técnicas e teóricas de fios e suturas, São Paulo: Roca, 1993, p. 9-18.

FOSSUM, T. W. Biomateriais, suturas e hemostasia. In: FOSSUM, T. W. Cirurgia de pequenos animais, São Paulo: Roca, 2002, Cap. 9, p. 48-64. FRIES, C. L.; BINNINGTON, A. G.; VALLE, V. E.; CONNOLLY, J. G.; HOLMBERG, D. V.; PENNOCK, P. Enterocystoplasty with cistectomy and subtotal intracapsular prostatectomy in the male dog. Veterinary Surgery, v. 20, n. 2, p. 104-112, 1991.

GONZALEZ, R.; BUSON, H.; REID, C.; REINBERG, Y. Seromuscular colocystoplasty lined with urothelium: experience with 16 patients. Urology, v. 45, p. 124, 1995.

HARARI, J. Cicatrização de ferimentos. In: HARARI, J. Cirurgia de pequenos animais. Porto Alegre: ARTMED, 1999, Cap. 4, p. 54-59.

HASTINGS, J. C.; VAN WINCKLE Jr.,W.; BARKER,E. The effect of suture materials on healing wounds of the bladder. Surgery gynecology \& Obstetrics, v. 140, n. 6, p. 933-937, 1975.

KAMINSKI, J. M.; KATZ, A. R.; RIVER, P.; WOODWARD, S. C. Urinary bladder calculus formation on sutures in rabbits, cats and dogs. Gynecology \& Obstetrics Surgery, v. 146, n. 3, p. 353-357, 1978.

\section{Conclusão}

Nas condições do experimento aqui apresentado, pode-se concluir que: a relação entre a reação inflamatória e fibrose demonstrou que o processo cicatricial ocorreu em menor período de tempo quando se empregou a poliglactina e que a mesma não apresentou reação inflamatória supurativa nem calcificação na linha de sutura, sendo, assim, mais eficiente que o ácido poliglicólico.
KROPP, B. P.; RIPPY, M. K.; BADYLAK, S. F. et al. Regenerative urinary bladder augmentation using smal intestinal submucosa: Urodinamic and histopathologic assessment in long-term canine bladder augmentation. The Journal Urology, v. 155, p. 2098-2104, 1996.

LAUS,J. L.; FERREIRA, A. L.; SOBREIA,L. F. R. Comparação entre os efeitos do dexon plus (ácido poliglicólico encapado) e do Maxon (poligliconato monofilamentar) sobre a cicatrização intestinal em enterorrafias ileais. Estudo experimental em cães (Canis familiaris). Brazilian journal veterinary research animal science, São Paulo, v. 32,n. 4, p. 241-245, 1995.

LIMA, S. V. C.; ARAÚJO,L. A. P.; MONTORO, M. e VILAR, F. O. O uso de intestino com mucosa removida para o aumento de bexigas pequenas e contraídas. Urodinâmica, v. 1, n. 1, jan./fev., 1996.

LIMA, S. V. C.; ARAÚJO,L. A. P.; MACIEL, A. e VILAR, F. O. A repercussão urodinâmica do uso de intestino desepitelizado para aumentar bexigas pequenas e contraídas. Urodinâmica, v. 1, n 1, jan./mar., 1998.

NANDI, S. K.; BOSE, P. K.; SENGUPTA, J.; CHATTERJEE, A. Change in the bladder capacity in goats after recontruction with pericardium - an experimental study. Indian Journal Animal HIth, v. 35, n. 2, p. 145-147, 1996.

PEACOCK, E. F. Wound repair. In: SLATTER, D. Textbook Small Animal Surgery, 3 ed. Philadelphia: W. B. Saunders Company, 1984, 2 v., v. 1 , p. 74.

POSTLETHWAIT, R. W.; WILLIGAN, D. A.; ULIN, A. W. Human tissue reaction to sutures. Annual Surgery, v. 181, p. 144-150, 1975.

PROPHET, E. B.; STEPHEN, J.E.; EDWARD, C.F. Laboratory methods in histotecnology, Armed Forces Institute of Pathology. American Registly of Pathology, Washington, 1994.

RODEHEAVER, G. T.; THACKER, J. G.; OWEN, J.; STRAUSS, M.; MASTERSON, T.; EDLICH, R. F. Knotting and hanling characteristics of coated synthetic absorbable sutures. Journal of Surgical Research, v. 35, n. 6, p. 525- 30, 1983.

SALLE, J. L. P.; FRAGA, J. C. S.; LUCIB, A. L.; LAMPERTZ, M.; JOBIM, G.; JOBIM, G.; PUTTEN, A. Seromuscular enterocystoplasty in dogs. Journal Urology, v. 144, parte 2, august, p. 454-456, 1990.

SHARMA, S. P., Urinary Bladder reconstruction using the intact caecum: an experimental study. The Indian Veterinary Journal, n. 74, p. 51-53, 1977.

SHARMA, S. P.; KHAN, A. A. Colocystoplasty for urinary bladder repair in buffalo calves. The Indian Veterinary Journal, v. 55, n. 4, april, 1978.

SLATTER, D. Sistema urinário. In: SLATTER, D. Manual de cirurgia de pequenos animais. 2 ed. São Paulo: Manole, 1998, 2 v., v. 2., Cap. 106, p. 1450-1461.

VORDERMARK, J. S.; IRBY, P. B.; SHEHATA, B. M.; BROWN, R. F. The affects of ileocystoplasty on the development of renal failure in a rat model 5/6 nephrectomy. Journal Urology, v. 148, august, 1992. 